\title{
The Role of 18F FDG PET/CT in Clinical Management of Breast Cancer; Additional Value Over Conventional Imaging Modalities
}

\author{
Mehrdad Bakhshayeshkaram, ${ }^{1,}$ Farahnaz Aghahosseini, ${ }^{2}$ Abtin Doroudinia, ${ }^{3}$ Soheyla Zahirifard, \\ Mojtaba Ansari, Maryam Hassanzad, and Hamid Reza Jamaat \\ ${ }^{1}$ Masih Daneshvari Hospital, Pediatric Respiratory Diseases Research Center, Tehran, Iran \\ ${ }^{2}$ Masih Daneshvari Hospital, Sahid Beheshti University of Medical Sciences, Tehran, Iran \\ ${ }^{3}$ Masih Daneshvari Hospital, Chronic Respiratory Diseases Research Center, Tehran, Iran \\ "Corresponding author: Mehrdad Bakhshayeshkaram, XAuthoraffiliactionX. E-mail: mehrdadbakhshayesh@yahoo.com \\ Received 2016 December 21; Accepted 2017 February 08.
}

\begin{abstract}
Background: The aim of the present study was to investigate the potential improvement in detection rate of nodal and distant metastasis and the resultant impact on clinical management by PET-based diagnostic work up in comparison with conventional imaging modalities in various clinical settings in patients with breast cancer.

Methods: Of a total 256 patients with breast cancer who were referred to PET/CT division, MasihDaneshvari Hospital between May 2013 and Mar. 2016, 123 cases were included. Definitely-positive PET/CT findings in primary site as well as N1 (level I, II ipsilateral axillary nodes), N2 (ipsilateral internal mammary), N3 (ipsilateral internal mammary+ level I, II ipsilateral axillary, ipsilateral level II axillary, ipsilateral supraclavicular node) and distant metastasis (distant node and viscera) and related impact on clinical management (upstaging or downstaging) were retrospectively identified from the report archive and then compared with the results of baseline imaging modalities including CT scan, sonography, bone scan, C-X ray, MRI, MRM and mammography obtained from patient medical records. The most typical imaging pattern was considered as the reference standard. Indeterminant and equivocal PET positive lesion were not included in the study.

Results: Fourteen (11.38\%) and 109 (88.62\%) out of 123 patients were referred for staging and restaging/recurrence/surveillance, respectively. Mean age were 51.42 (34 - 62) and 52.66 (29 - 79) for staging and restaging groups, respectively. In staging group, 18 F FDG PET/CT recognized definitely positive additional nodal involvement in $21.4 \%$ of patients all of them related to the supraclavicular level and hence result in change $\mathrm{N}$ staging to $\mathrm{N} 3$. Additional metastatic lesions on a per lesion-basis and a per organ-basis were identified as $28.6 \%$ and $14.3 \%$, repsectively. A significant change in patient management occurred in $42.9 \%$ (35.7\% upstaging, $7.2 \%$ down staging). In restaging group, locoregional detection rate in biopsy proved recurrence was $100 \%$. Additional nodal detection and the resultant change in N staging were $29.6 \%$ and $28.7 \%$, respectively. Supraclavicular node detection contribute in $53.12 \%$ of overall change in $\mathrm{N}$ staging followed by axillary node and internal mammary equally (15.62\%). Additional metastatic lesions on a per lesionbasis and per organ-basis were identified in $53.2 \%$ and $44 \%$, respectively. Ultimate change in patients' management was found in $42.9 \%$.

Conclusions: 18 F FDG PET/CT is a valuable imaging tool in initial and subsequent treatment strategy in patients with breast cancer by providing additional nodal and distant metastasis and may have the promising future potential to be as the imaging modality of choice for accurate assessment of disease extension in breast cancer in routine clinical practice.
\end{abstract}

Keywords: 18F FDG PET/CT, Breast Cancer, Conventional Imaging, Clinical Management

This is an abstract presented in the 33rd Iranian congress of radiology (ICR) and the 15th congress of Iranian radiographic science association (IRSA) 